\title{
Respuesta carta al editor titulada: Lupus Eritematoso Sistémico Infantil: características neuropsiquiátricas y factores pronóstico en su transición a la adultez
}

\author{
Response to the letter of editor entitled: Childhood-Onset Systemic Lupus \\ Erythematosus: neuropsychiatric features and prognostic factors in their transition \\ into adulthood
}

\author{
Paula Valenzuela ${ }^{\mathrm{a}}$, Mabel Ladino ${ }^{\mathrm{b}, \mathrm{c}}$
}

aUnidad de Emergencia Infantil, Hospital Félix Bulnes. Santiago, Chile

bUnidad de Reumatología Pediátrica, Hospital San Juan de Dios. Santiago, Chile

'Departamento de Pediatría, Universidad de Chile. Santiago, Chile

\section{Sr. Lector,}

Luego de leer su carta al Editor, en atención a nuestro artículo, debemos agradecer los comentarios expuestas por su autor.

Como usted describe, las manifestaciones neuropsiquiátricas no son las más comunes al momento diagnóstico del LES Infantil, pero son una causa importante de morbilidad y complicaciones tanto en la etapa pediátrica como en la adultez. En nuestro estudio 4 de 31 pacientes evaluados presentaron alguna neuropsiquiátrica durante la etapa pediátrica (2: Síndrome Convulsivo y 2: Accidentes Vasculares) y no se presentaron pacientes con corea tanto en la etapa pediátrica como en la evolución a la etapa adulta.

Es importante destacar que según nuestro estudio las manifestaciones más frecuentes al momento del diagnostico fueron la artritis, seguido de exantema malar y nefropatía, lo que coincide con otros estudios realizados en población pediátrica.

Nuestro estudio no tuvo como objetivo correlacionar el tipo de manifestación clínica al debut del diagnóstico (como por ej. las manifestaciones neurológicas) con una peor evolución en la etapa adulta, como lo sugiere se sugiere en la carta al editor, pero recibimos la sugerencia con gran interés para una próxima publicación.

Esperamos que nuestro articulo sirva para incentivar el estudio y conocimiento del LES infantil y su evolución a adultez en la población sudamericana.

Saludamos cordialmente a los autores de esta carta al editor. 